\title{
Editorial
}

\section{Selected Papers from ISEF-2015 Conference}

The origin of ISEF, the International Symposium on Electromagnetic Fields in Mechatronics, Electrical and Electronic Engineering, dates back to the year 1974 in Poland: seventeen editions of the symposium in more than forty years of history are a nice age in such modern and rapidly developing area of science. Over the years, ISEF has gained a prominent positions in the electromagnetic community because it gathers a relatively small, but strongly linked community of specialists in applied electromagnetism. This is perhaps the key to further development of the Symposium, whose first objective is fostering innovative applications of engineering science in the broad area of fields.

ISEF-2015 was successfully organized at the Technical University of Valencia (Spain) thanks to the support of Professor Manuel Pineda Sanchez and his co-operators. The Symposium was the meeting point of researchers, engineers and designers interested in modelling, simulation, measurements and applications of electromagnetic fields. New emerging fields of research, i.e. bioelectromagnetism and biomedical problems, have been the subject of a special session, in addition to more classical topics like e.g. noise in electrical machines, propagation of radiowaves, microelectromechanical systems. Moreover, a panel session focusing on recent advances in automated optimal design was organized with the format of an interactive forum: after an overview from the panellists, hot topics were discussed by the audience.

Very many computational methods have been developed, but not all of them appear convenient enough for the use in practical engineering. In fact, technical and industrial challenges set some principal requirements and criteria for good computational methods. In this respect, the main task of a researcher consists of reducing the still intractable problems of field analysis and synthesis to the search for cost-effective approximate solutions based on friendly tools. This is one of the most important messages which came out of the Symposium.

Specifically, 188 papers out of 227 submitted papers have been accepted for presentation at ISEF2015, involving authors from 29 countries. The collection of twenty papers, selected after a peer review procedure for this special issue of IJAEM, covers the main topics discussed at the conference. The editors would like to thank all the authors for their valuable contributions and, in particular, the editor-in-chief of the Journal for the opportunity of addressing a greater international audience.

Paolo Di Barba ${ }^{\mathrm{a}}$, Slawomir Wiak ${ }^{\mathrm{b}}$

${ }^{a}$ University of Pavia, Pavia, Italy

${ }^{a}$ Lodz University of Technology, Lodz, Poland

Guest editors 